\title{
Single locus inheritance and joint segregation analysis of minisatellite (VNTR) DNA loci in brown trout (Salmo trutta L.)
}

\author{
PAULO A. PRODÖHL*, JOHN B. TAGGART† \& ANDREW FERGUSON \\ School of Biology and Biochemistry, Queen's University of Belfast, Belfast BT7 1NN, Northern Ireland, U.K. and \\ $\dagger$ Department of Biological and Molecular Sciences, School of Natural Sciences, University of Stirling, Stirling FK9 4LA, \\ Scotland, U.K.
}

\begin{abstract}
Eleven minisatellite (VNTR) DNA loci were examined for Mendelian inheritance and possible linkage relationships in a brown trout family (54 offspring). Analyses of the full progeny set (males and females combined) showed no statistically significant departures from Mendelian expectations at any locus, and no new mutations were observed. However, at one locus, Str-A9, differential segregation $(P<0.001)$ of parental alleles into male and female progeny was recorded. This is indicative of tight linkage between Str-A9 and the sex determining region in the brown trout, and confirms the male as the heterogametic sex in this species. Joint segregation analyses of 83 different pairwise comparisons of loci revealed nonrandom assortment in only two instances, i.e. both male and female comparisons of Str-A22/1 with Str-A22/2. These findings illustrate the importance of inheritance studies in clarifying the genetic basis underlying minisatellite DNA polymorphisms prior to their use as individual, familial or population markers.
\end{abstract}

Keywords: brown trout, inheritance, linkage, minisatellite DNA, Salmo trutta, VNTR.

\section{Introduction}

Most salmonid fishes are of significant economic and recreational importance. As a consequence, extensive studies concerning the population biology and genetics of many of these species have been undertaken (e.g. Ferguson \& Taggart, 1991; Ryman, 1991; Shaklee et al., 1991; Utter, 1991; Verspoor, 1994). Furthermore, being a relatively recent tetraploid derivative group (Ohno et al., 1969), the salmonids have attracted considerable attention in the study of evolution by gene duplication. The advent of gel electrophoresis (in the $1960 \mathrm{~s}$ ), which allows the indirect assessment of genetic variability through the study of protein polymorphisms (Ferguson, 1980), has greatly benefited these lines of enquiry. However, although a considerable number of polymorphic systems have been detected in most salmonid species (e.g. Utter, 1991), usually only a few alleles segregate at a locus in any particular population and heterozygosity levels are generally low. As a result, detailed studies concerning population genetics and evolutionary aspects of salmonid biology have been somewhat restricted.

*Correspondence.
More recently, minisatellite DNA fingerprinting studies of salmonids have revealed an alternative source of highly polymorphic markers (e.g. Fields et al., 1989; Taggart \& Ferguson, 1990a; Bentzen et al., 1991). Due to their complexity, however, multilocus profiles are only of limited value for salmonid studies, especially at the population level (Taggart \& Ferguson, 1990a; Prodöhl et al., 1992). Locus-specific minisatellite (VNTR) DNA probes provide a more practical approach to the detection and analysis of these highly variable regions of nuclear DNA. Unlike 'universal' multilocus DNA probes, however, single locus minisatellite probes (SLPs) are largely species/genus specific and thus must be individually isolated for each organism under investigation. While many SLPs have been produced for humans (e.g. Nakamura et al., 1987; Wong et al., 1987; Armour et al., 1990), the specialized nature of the molecular cloning procedures required to isolate them has been a major factor in the relatively limited application of this new technology to other eukaryotes. Nevertheless, SLPs have been recently reported for a number of organisms; mainly other mammals (e.g. Kominami et al., 1988; Kelly et al., 1989; Coppieters et al., 1990), and various bird species (e.g. Bruford \& Burke, 1991; Burke et al., 1991; Hanotte et al., 1991b). 
To date, reports of SLP isolation among fish species have been mainly confined to salmonids, specifically Atlantic salmon, Salmo salar L. (Taggart \& Ferguson, 1990b; Bentzen et al., 1991), and brown trout, Salmo trutta L., (Prodöhl et al., 1994). Similar probes have also been isolated for tilapia (Bentzen et al., 1991). These preliminary studies have revealed the presence of a much larger number of alleles at minisatellite loci in comparison with protein coding loci. A battery of such SLPs, with a range of heterozygosities, would prove invaluable in many aspects of salmonid research by providing population, familial, individual and chromosomal markers.

It is essential that both the genetic basis underlying observed variation detectable with SLPs and possible linkage relationships among minisatellite loci be established prior to their more widespread use. This is of particular importance with regard to salmonids, where occasional forms of aberrant segregation at protein coding loci have been observed, these being considered to reflect residual tetrasomy within the salmonid genome (May et al., 1979; Wright et al., 1983). In this paper we describe a panel of 11 SLPs which detect single locus polymorphisms in the brown trout. Both single locus and joint segregation inheritance studies (including sex linkage) involving all loci are detailed.

\section{Materials and methods}

\section{Samples}

Brown trout crosses were performed in November and December 1989 using local fish farm broodstock. Three families were reared to a size in which the sex of the offspring could be determined by gonadal inspection. These comprised one large family of which 48 individuals were sexed (F89/1, no. progeny $=54$; due to their small size sex could not be confidently determined for six offspring) and two smaller families (F89/ $2, n=15 ; \mathrm{F} 89 / 3, \mathrm{n}=23$ ). Population samples were available from an ongoing series of investigations being undertaken within our laboratory and represented geographically widely separated stocks (Ireland and Iceland). In all cases, tissues (muscle) were stored frozen $\left(-20^{\circ} \mathrm{C}\right)$ or in 99 per cent ethanol $\left(+4^{\circ} \mathrm{C}\right)$ until required.

\section{Routine screening conditions}

Total genomic DNA was isolated using a simplified phenol / chloroform methodology (Taggart et al., 1992). Standard protocols for gel electrophoresis ( $3 \mu \mathrm{g}$ Pall digested DNA/sample), Southern blotting (onto Hybond $\mathrm{N}$ membrane) and isotopic labelling of probe (random priming) were followed. Prehybridization and hybridization conditions were $1.5 \times \operatorname{SSPE}(0.27 \mathrm{M}$ $\mathrm{NaCl}, 15 \mathrm{~mm} \mathrm{NaH} \mathrm{PO}_{4}, 1.5 \mathrm{~mm}$ EDTA $\mathrm{Na}_{2}$ salt; $\mathrm{pH}$ 7.7), 0.5 per cent dried milk, 1 per cent sodium dodecyl sulphate (SDS), 6 per cent polyethylene glycol (PEG) at $65^{\circ} \mathrm{C}$ (Dalgleish, 1987). Sonicated brown trout genomic DNA $\left(10 \mu \mathrm{g} \mathrm{mL}^{-1}\right)$ was added, as competitor, to the hybridization mix. Following overnight hybridization, membranes were washed to high stringency $\left(0.5-0.1 \times \mathrm{SSC}, 0.1\right.$ per cent $\left.\mathrm{SDS}, 65^{\circ} \mathrm{C}, 2 \times 30 \mathrm{~min}\right)$ and autoradiographed for $2-4$ days at $-70^{\circ} \mathrm{C}$ in the presence of intensifying screens $[2 \times \mathrm{SSC}=0.3 \mathrm{M} \mathrm{NaCl}$, $0.03 \mathrm{M} \mathrm{Na}_{3}$ citrate; $\mathrm{pH} 7.0$ ].

\section{Probe / locus nomenclature}

Following a system previously adopted by others (Bentzen et al., 1991; Hanotte et al., 1991a), each SLP is identified by initial letters from the species binomial, followed by a unique (usually original clone) number. We use a prefix ' $p$ ' when referring to the probe as distinct from the locus it detects. To avoid duplication of names (arising from different laboratories isolating SLPs from the same species) we suggest the inclusion of an additional laboratory identifier, i.e. a single capital letter preceding the number. Thus, probes isolated by our group from brown trout have been designated $\mathrm{p} S t r-\mathrm{A} n$ and from Atlantic salmon (S. salar), pSsa-A $n$. Where more than one locus may be detected by an original recombinant (see below), additional numeric locus designators are used following a 'slash' separator e.g. Ssa-A45/1 and Ssa-A45/2. Similarly, probes later derived to detect each locus separately have been named $\mathrm{pSsa}-\mathrm{A} 45 / 1$ and $\mathrm{pSsa}-\mathrm{A} 45 / 2$, respectively, thereby signifying their common origin. The term 'allele' is used in a general context, referring to entire DNA fragments resolved by each probe (i.e. minisatellite repeat region(s) and any adjacent DNA). Individual fragments are assumed to be single 'alleles' although in some cases they may represent groups of isoalleles. Specific alleles are described by simple alphabetic notation.

\section{Single locus probes}

The methodology for, and rationale behind, the isolation of five probes ( $\mathrm{p}$ Str-A1, $\mathrm{p}$ Str-A3, $\mathrm{p}$ Str-A5, $\mathrm{p}$ Str-A9 and $\mathrm{p} S t r-\mathrm{A} 22$ ) from a brown trout DNA phagemid library have been detailed elsewhere (Prodöhl et al., 1994). It has since been found that Southern blots probed with $\mathrm{p} S t r-\mathrm{A} 22$ and washed at lower stringency than normal $(0.3 \times \mathrm{SSC}$ vs. $0.1 \times \mathrm{SSC})$ resolved an additional polymorphic locus. Further investigation revealed that this probe insert $(\approx 1500 \mathrm{bp}-\mathrm{Mbol}$ fragment) contained an internal Pall site yielding two frag- 
ments of $800 \mathrm{bp}$ and $700 \mathrm{bp}$ in size. Each fragment was subsequently subcloned into pBluescript II phagemid (Stratagene) giving probes pStr-A22/1 and pStr-A22/2, respectively, each probe detecting a different locus with $P a l$ digested genomic DNA. Additionally, five Atlantic salmon derived SLPs were surveyed. Details of the isolation of four of these probes, from an EMBL3 partial library, have been given previously (Taggart \& Ferguson, 1990b; Taggart et al., 1994). These probes, originally designated $3.15 .33,3.15 .34,3.15 .45$ and 3.15 .60 have been renamed pSsa-A33, pSsa-A34, $\mathrm{p} S s a-\mathrm{A} 45 / 1$ and $\mathrm{p} S s a-\mathrm{A} 60$, respectively. The original 3.15.45 EMBL3 recombinant yielded three Sau3A I insert fragments, only one of which detected the variable locus referred to by Taggart \& Ferguson (1990b). A second fragment, pSsa-A45/2, has since been found to detect apparent minisatellite DNA variation in Atlantic salmon, and was also used in this study. ( $\mathrm{pSsa}-\mathrm{A} 34, \mathrm{pSsa}-\mathrm{A} 45 / 1$ and $\mathrm{pSsa}-\mathrm{A} 45 / 2$ have since been subcloned into pBluescript II phagemid constructs.)

\section{Inheritance analyses}

Goodness of fit $\chi^{2}$ statistics were employed to test for departures from Mendelian segregation of alleles at each minisatellite DNA locus. Separate analyses were carried out on male only, female only, and combined progeny in order to screen for possible sex linkage effects. In conformance with previous joint segregation analyses of salmonids (e.g. May et al., 1979; Taggart \& Ferguson, 1984) the symbols and statistics of Mather (1951) were employed, where for a given test cross $A A B B \times A A^{\prime} B B^{\prime}$ resultant offspring were grouped as follows:

$a_{1}=$ observed progeny $[A A B B]$,

$a_{2}=$ observed progeny $\left[A A B B^{\prime}\right]$,

$a_{3}=$ observed progeny $\left[A A^{\prime} B B\right]$ and

$a_{4}=$ observed progeny $\left[A A^{\prime} B B^{\prime}\right]$.

This gives the chi-square statistic for independent segregation of the alleles at the two loci,

$\chi_{\mathrm{AB}}^{2}=\left(a_{1}-a_{2}-a_{3}+a_{4}\right)^{2} / N($ d.f. $=1)$,

and the recombination fraction or fraction of nonparental genotypes (assuming the larger class, $\left(a_{1}+a_{4}\right)$ or $\left(a_{2}+a_{3}\right)$, represents the parental genotypes $)$,

$r=\left(a_{2}+a_{3}\right) / N$ or $\left(a_{1}+a_{4}\right) / N$,

respectively.

\section{Results}

With only one exception (i.e. pSsa-A60, where occasionally no bands were observed), each probe examined resolved either a single- or double-banded phenotype per individual. Some typical autoradiographs, Fig. 1, illustrate the routine standard of resolution that was achieved and also demonstrate inheritance. Both the extent and the pattern of polymorphism detected by each probe from population data were consistent with those expected for minisatellite DNA length variation at a single locus.

A summary of probe characteristics, including preliminary population data where available, is given in Table 1 . All loci were found to be at least moderately variable (i.e. 4-13 discrete allele classes; lowest mean heterozygosity 21 per cent). Interestingly, most allele classes were observed in the majority of populations surveyed, with no high frequency (i.e. $>0.3$ ) population diagnostic bands apparent.

Fortuitously, the largest family (F89/1) proved to be the most informative of the three sexed crosses available, with the female parent being heterozygous at all 11 loci and the male parent at eight loci. Str-A1 and Ssa-A45/1 were both homozygous in the male parent, while no bands were detectable with pSsa-A60. (This latter probe generally detects very small alleles in brown trout (1-2 kb), the 'null' genotype most likely reflecting particularly small alleles in this individual. DNA fragments $<500$ bp were run off the end of the gel.) With at least two expected progeny types per locus, it was possible to test for Mendelian (disomic) segregation of alleles at all loci and for possible sex associations in the majority of cases from this single family.

Analyses of the full progeny set (i.e. 54 offspring) revealed no statistically significant departure from Mendelian expectations at any locus (detailed in Table 2 ). This was also the case for sex-segregated progeny at 10 of the 11 loci. However, highly statistically significant differences were observed at Str-A9, which revealed differential segregation of paternal alleles into male and female progeny (Fig 1b). To confirm this finding the two smaller families were also screened with pStr-A9. Similar sex associations were observed in all cases (Table 3 ). Only two presumed recombinants were found out of 86 progeny scored (recombination frequency $=0.023$ ) suggesting tight linkage between Str-A9 and the sex determining region in brown trout. (Although unlikely, possible gender mistyping of these two individuals cannot be dismissed at present.) While it was not possible to test for male sex association with three probes in family 89/1, this was examined for StrA1 and Ssa-A45/1 (though with more limited progeny) in families F89/2 and F89/3. The two male parents were informative (i.e. heterozygous) for both these loci. No evidence of sex linkage was apparent for either locus (data not shown). Ssa-A60 was found to be noninformative (i.e. both males homozygous) in both of these families. 
(a) pStr-A3

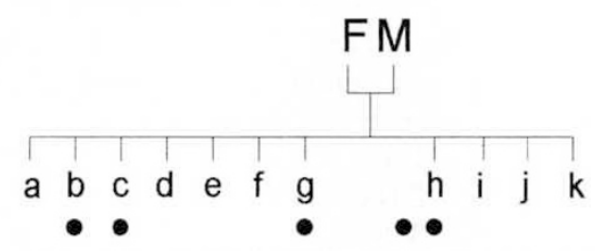

$6.6-$

(kb)

$5.6-$
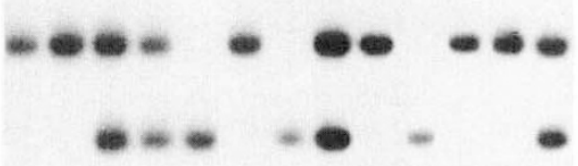

$6.6-$

(kb)

$5.6-$

$4.8-$ (b) pStr-A9

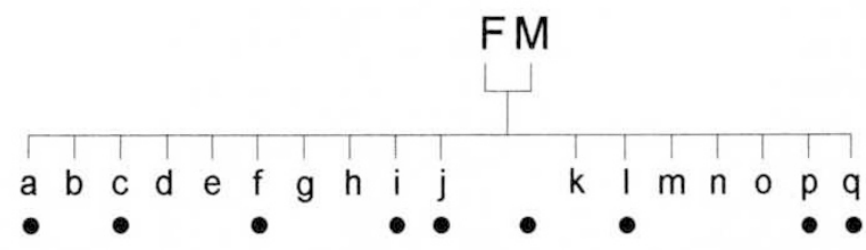

\section{$3.5-$}

(c) pStr-A22/1

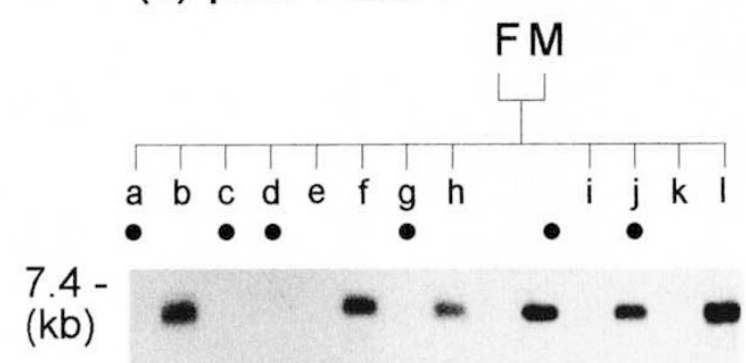

$3.5-$

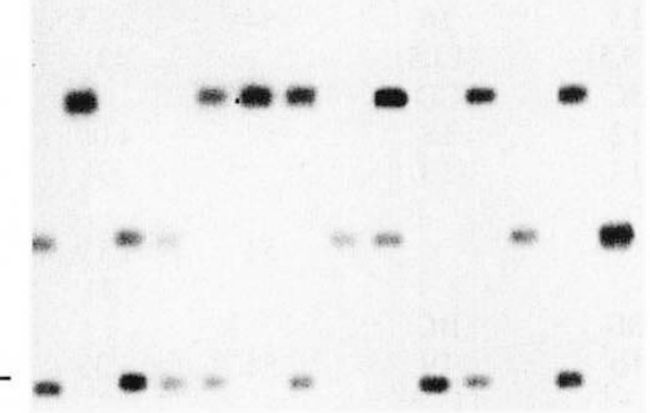

\section{$2.3-$}

(d) pStr-A22/2

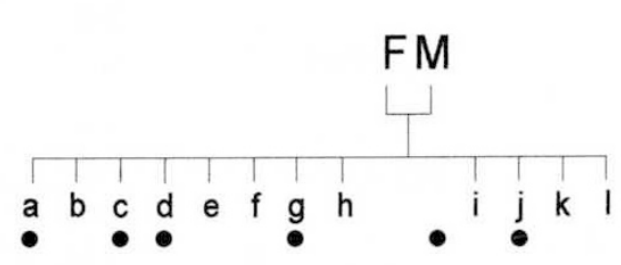

$3.5-$

$(\mathrm{kb})$

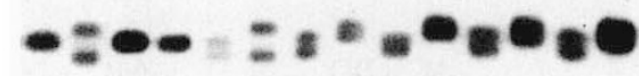

$2.3-$

Fig. 1 Examples of DNA profiles detected with brown trout minisatellite DNA single locus probes. In each case a brown trout family comprising male parent $(\mathrm{M})$, female parent $(\mathrm{F})$ and 11 to 17 progeny are shown. Male progeny are denoted by $(\bullet)$. DNA fragment size scale is given in kilobase pairs $(\mathrm{kb})$. 
Table 1 Summary of the basic characteristics of 11 locus-specific minisatellite DNA probes. With the exceptions of pSsa-A33 \& A60, where less than 30 individuals have been typed, allele statistics are based on 490 brown trout sampled from eight different populations from Ireland and Iceland

\begin{tabular}{lccccc}
\hline $\begin{array}{l}\text { Probe } \\
\text { name }\end{array}$ & $\begin{array}{c}\text { Probe } \\
\text { size }(\mathrm{kb})\end{array}$ & $\begin{array}{c}\text { Allele size } \\
\text { range }(\mathrm{kb})\end{array}$ & $\begin{array}{c}\text { Total no. } \\
\text { alleles }\end{array}$ & $\begin{array}{c}\text { No. alleles } \\
\text { per population }\end{array}$ & $\begin{array}{c}\text { Mean observed } \\
\text { heterozygosity }(\%)\end{array}$ \\
\hline $\mathrm{p}$ Str-A1 & 1.9 & $2.0-3.0$ & 6 & $1-6$ & 59 \\
$\mathrm{p}$ Str-A3 & 4.4 & $2.3-7.4$ & 11 & $2-11$ & 66 \\
$\mathrm{p}$ Str-A5 & 4.3 & $3.5-7.5$ & 9 & $3-8$ & 62 \\
$\mathrm{p}$ Str-A9 & 1.8 & $2.0-21.0$ & 13 & $4-7$ & 51 \\
$\mathrm{p}$ Str-A22/1 & 0.7 & $1.0-7.4$ & 9 & $6-8$ & 69 \\
$\mathrm{p}$ Str-A22/2 & 0.8 & $2.4-3.8$ & 6 & $1-4$ & - \\
$\mathrm{p}$ Ssa-A33 & 17.0 & $15.5-23.0$ & 4 & - & 56 \\
$\mathrm{p}$ Ssa-A34 & 3.8 & $2.5-4.9$ & 10 & $2-8$ & 53 \\
$\mathrm{p}$ Ssa-A45/1 & 4.2 & $4.6-14.8$ & 13 & $2-6$ & 48 \\
$\mathrm{p}$ Ssa-A45/2 & 2.9 & $4.3-7.0$ & 6 & $2-6$ & - \\
$\mathrm{p}$ Ssa-A60 & 10.5 & $1.0-2.1$ & 4 & - & - \\
\hline
\end{tabular}

Table 2 Summary of single locus analyses for the 11 minisatellites examined, including $\chi^{2}$ test for Mendelian segregation of alleles in the brown trout family F89/1

\begin{tabular}{|c|c|c|c|c|c|c|c|c|}
\hline & $\begin{array}{l}\text { Parental genotype } \\
\text { female } \times \text { male }\end{array}$ & & $\begin{array}{l}\text { Progeny } \\
\text { observed }\end{array}$ & $\begin{array}{l}\text { notype } \\
\text { spected) }\end{array}$ & & Total & $\begin{array}{l}\chi^{2} \\
\text { (d.f.) }\end{array}$ & $P$ \\
\hline \multirow[t]{2}{*}{ Str-A1 } & $\mathrm{BC} \times \mathrm{AA}$ & $\mathrm{AB}$ & $\mathrm{AC}$ & & & & & \\
\hline & & $\begin{array}{c}33 \\
(27)\end{array}$ & $\begin{array}{c}21 \\
(27)\end{array}$ & & & 54 & $\begin{array}{c}2.67 \\
(1)\end{array}$ & 0.102 \\
\hline \multirow[t]{2}{*}{ Str-A3 } & $\mathrm{BC} \times \mathrm{AB}$ & $\mathrm{AB}$ & $\mathrm{AC}$ & BB & $\mathrm{BC}$ & & & \\
\hline & & $\begin{array}{c}14 \\
(13.25)\end{array}$ & $\begin{array}{c}12 \\
(13.25)\end{array}$ & $\begin{array}{c}12 \\
(13.25)\end{array}$ & $\begin{array}{c}15 \\
(13.25)\end{array}$ & 53 & $\begin{array}{c}0.51 \\
(3)\end{array}$ & 0.917 \\
\hline \multirow[t]{2}{*}{ Str-A5 } & $\mathrm{BC} \times \mathrm{AB}$ & $\mathrm{AB}$ & $\mathrm{AC}$ & BB & $\mathrm{BC}$ & & & \\
\hline & & $\begin{array}{c}14 \\
(13.5)\end{array}$ & $\begin{array}{c}12 \\
(13.5)\end{array}$ & $\begin{array}{c}13 \\
(13.5)\end{array}$ & $\begin{array}{c}15 \\
(13.5)\end{array}$ & 54 & $\begin{array}{l}0.37 \\
(3)\end{array}$ & 0.946 \\
\hline \multirow[t]{2}{*}{ Str-A9 } & $\mathrm{BC} \times \mathrm{AD}$ & $\mathrm{AB}$ & $\mathrm{AC}$ & $\mathrm{BD}$ & $\mathrm{CD}$ & & & \\
\hline & & $\begin{array}{c}12 \\
(13.5)\end{array}$ & $\begin{array}{c}15 \\
(13.5)\end{array}$ & $\begin{array}{c}12 \\
(13.5)\end{array}$ & $\begin{array}{c}15 \\
(13.5)\end{array}$ & 54 & $\begin{array}{c}0.67 \\
(3)\end{array}$ & 0.881 \\
\hline \multirow[t]{2}{*}{ Str-A22/1 } & $\mathrm{BC} \times \mathrm{AD}$ & $\mathrm{AB}$ & $\mathrm{AC}$ & $\mathrm{BD}$ & $\mathrm{CD}$ & & & \\
\hline & & $\begin{array}{c}17 \\
(13.5)\end{array}$ & $\begin{array}{c}12 \\
(13.5)\end{array}$ & $\begin{array}{c}12 \\
(13.5)\end{array}$ & $\begin{array}{c}13 \\
(13.5)\end{array}$ & 54 & $\begin{array}{l}1.26 \\
(3)\end{array}$ & 0.739 \\
\hline \multirow[t]{2}{*}{ Str-A22/2 } & $\mathrm{BC} \times \mathrm{AB}$ & $\mathrm{AB}$ & $\mathrm{AC}$ & $\mathrm{BB}$ & $\mathrm{BC}$ & & & \\
\hline & & $\begin{array}{c}12 \\
(13.5)\end{array}$ & $\begin{array}{c}17 \\
(13.5)\end{array}$ & $\begin{array}{c}13 \\
(13.5)\end{array}$ & $\begin{array}{c}12 \\
(13.5)\end{array}$ & 54 & $\begin{array}{l}1.26 \\
(3)\end{array}$ & 0.739 \\
\hline \multirow[t]{2}{*}{ Ssa-A33 } & $\mathrm{AB} \times \mathrm{BC}$ & $\mathrm{AB}$ & $\mathrm{AC}$ & $\mathrm{BB}$ & $\mathrm{BC}$ & & & \\
\hline & & $\begin{array}{c}9 \\
(13.5)\end{array}$ & $\begin{array}{c}14 \\
(13.5)\end{array}$ & $\begin{array}{c}15 \\
(13.5)\end{array}$ & $\begin{array}{c}16 \\
(13.5)\end{array}$ & 54 & $\begin{array}{c}2.15 \\
(3)\end{array}$ & 0.542 \\
\hline \multirow[t]{2}{*}{ Ssa-A34 } & $\mathrm{AC} \times \mathrm{BD}$ & $\mathrm{AB}$ & $\mathrm{AD}$ & $\mathrm{BC}$ & $\mathrm{CD}$ & & & \\
\hline & & $\begin{array}{c}8 \\
(13)\end{array}$ & $\begin{array}{c}14 \\
(13)\end{array}$ & $\begin{array}{c}12 \\
(13)\end{array}$ & $\begin{array}{c}18 \\
(13)\end{array}$ & 52 & $\begin{array}{l}4.00 \\
(3)\end{array}$ & 0.261 \\
\hline \multirow[t]{2}{*}{ Ssa-A45/1 } & $\mathrm{AB} \times \mathrm{BB}$ & $\mathrm{AB}$ & $\mathrm{BB}$ & & & & & \\
\hline & & $\begin{array}{c}23 \\
(27)\end{array}$ & $\begin{array}{c}31 \\
(27)\end{array}$ & & & 54 & $\begin{array}{l}1.19 \\
(1)\end{array}$ & 0.276 \\
\hline \multirow[t]{2}{*}{ Ssa-A45/2 } & $\mathrm{BC} \times \mathrm{AB}$ & $\mathrm{AB}$ & $\mathrm{AC}$ & BB & BC & & & \\
\hline & & $\begin{array}{c}13 \\
(13.5)\end{array}$ & $\begin{array}{c}12 \\
(13.5)\end{array}$ & $\begin{array}{c}10 \\
(13.5)\end{array}$ & $\begin{array}{c}19 \\
(13.5)\end{array}$ & 54 & $\begin{array}{c}3.33 \\
(3)\end{array}$ & 0.343 \\
\hline \multirow[t]{2}{*}{ Ssa-A60 } & $\mathrm{AB} \times \mathrm{n}^{*}$ & An & $\mathrm{Bn}$ & & & & & \\
\hline & & $\begin{array}{c}24 \\
(27)\end{array}$ & $\begin{array}{c}30 \\
(27)\end{array}$ & & & 54 & $\begin{array}{l}0.67 \\
(1)\end{array}$ & 0.414 \\
\hline
\end{tabular}

${ }^{*} \mathrm{n}=$ null allele. 
Under the electrophoretic conditions employed no nonparental allele was observed among progeny at any of the minisatellite loci, indicative of a relatively high germline stability for these loci. Based on 11 loci examined among 54 offspring, and additional screening of families $89 / 2$ and $89 / 3$, the maximum combined mutation rate at these minisatellite loci must be less than $0.003 /$ allele/generation (95 per cent confidence maximum).

Family F89/1 proved equally informative for joint segregation analyses. A total of 83 different pairwise comparisons of loci was possible (Table 4). Joint

Table 3 Single locus inheritance analyses for Str-A9 in three brown trout families. Both combined (male and females) and sex segregated data are presented with $\chi^{2}$ tests for conformance with Mendelian expectations

\begin{tabular}{|c|c|c|c|c|c|c|c|c|}
\hline \multirow{2}{*}{$\begin{array}{l}\text { Family } \\
\text { F89/1 } \\
\text { combined }\end{array}$} & $\begin{array}{c}\begin{array}{c}\text { Parental genotype } \\
\text { female } \times \text { male }\end{array} \\
\mathrm{BC} \times \mathrm{AD}\end{array}$ & \multicolumn{4}{|c|}{$\begin{array}{c}\text { Progeny genotype } \\
\text { observed/(expected) }\end{array}$} & \multirow{2}{*}{$\begin{array}{c}\text { Total } \\
54\end{array}$} & \multirow{2}{*}{$\begin{array}{c}\begin{array}{c}\chi^{2} \\
\text { (d.f.) }\end{array} \\
0.67 \\
(3)\end{array}$} & \multirow{2}{*}{$\begin{array}{c}P \\
0.881\end{array}$} \\
\hline & $\mathrm{BC} \times \mathrm{AD}$ & $\begin{array}{c}\mathrm{AB} \\
12 \\
(13.5)\end{array}$ & $\begin{array}{c}\mathrm{AC} \\
15 \\
(13.5)\end{array}$ & $\begin{array}{c}\text { BD } \\
12 \\
(13.5)\end{array}$ & $\begin{array}{c}C D \\
15 \\
(13.5)\end{array}$ & & & \\
\hline female & & $\begin{array}{c}9 \\
(6.25)\end{array}$ & $\begin{array}{c}15 \\
(6.25)\end{array}$ & $\begin{array}{c}1 \\
(6.25)\end{array}$ & $\begin{array}{c}0 \\
(6.25)\end{array}$ & 25 & $\begin{array}{c}24.12 \\
(3)\end{array}$ & $<0.001$ \\
\hline male & & $\begin{array}{c}0 \\
(5.75)\end{array}$ & $\begin{array}{c}0 \\
(5.75)\end{array}$ & $\begin{array}{c}9 \\
(5.75)\end{array}$ & $\begin{array}{c}14 \\
(5.75)\end{array}$ & 23 & $\begin{array}{c}25.17 \\
(3)\end{array}$ & $<0.001$ \\
\hline $\begin{array}{l}\mathrm{F} 89 / 2 \\
\quad \text { combined }\end{array}$ & $\mathrm{AB} \times \mathrm{AC}$ & $\begin{array}{c}\mathrm{AA} \\
6 \\
(5.75)\end{array}$ & $\begin{array}{c}\mathrm{AB} \\
4 \\
(5.75)\end{array}$ & $\begin{array}{c}\mathrm{AC} \\
6 \\
(5.75)\end{array}$ & $\begin{array}{c}\mathrm{BC} \\
7 \\
(5.75)\end{array}$ & 23 & $\begin{array}{l}0.83 \\
(3)\end{array}$ & 0.843 \\
\hline female & & $\begin{array}{c}6 \\
(3.25)\end{array}$ & $\begin{array}{c}4 \\
(3.25)\end{array}$ & $\begin{array}{c}1 \\
(3.25)\end{array}$ & $\begin{array}{c}0 \\
(3.25)\end{array}$ & 11 & $\begin{array}{l}8.27 \\
(3)\end{array}$ & 0.041 \\
\hline male & & $\begin{array}{c}0 \\
(2.5)\end{array}$ & $\begin{array}{c}0 \\
(2.5)\end{array}$ & $\begin{array}{c}5 \\
(2.5)\end{array}$ & $\begin{array}{c}7 \\
(2.5)\end{array}$ & 12 & $\begin{array}{c}2.67 \\
(3)\end{array}$ & 0.005 \\
\hline $\begin{array}{l}\mathrm{F} 89 / 3 \\
\quad \text { combined }\end{array}$ & $\mathrm{BC} \times \mathrm{AB}$ & $\begin{array}{c}\mathrm{AB} \\
2 \\
(3.75)\end{array}$ & $\begin{array}{c}\mathrm{AC} \\
5 \\
(3.75)\end{array}$ & $\begin{array}{c}\text { BB } \\
5 \\
(3.75)\end{array}$ & $\begin{array}{c}\mathrm{BC} \\
3 \\
(3.75)\end{array}$ & 15 & $\begin{array}{l}1.80 \\
(3)\end{array}$ & 0.615 \\
\hline female & & $\begin{array}{r}0 \\
(2)\end{array}$ & $\begin{array}{r}0 \\
(2)\end{array}$ & $\begin{array}{r}5 \\
(2)\end{array}$ & $\begin{array}{r}3 \\
(2)\end{array}$ & 8 & $\begin{array}{l}9.00 \\
(3)\end{array}$ & 0.029 \\
\hline male & & $\begin{array}{c}2 \\
(1.75)\end{array}$ & $\begin{array}{c}5 \\
(1.75)\end{array}$ & $\begin{array}{c}0 \\
(1.75)\end{array}$ & $\begin{array}{c}0 \\
(1.75)\end{array}$ & 7 & $\begin{array}{l}9.57 \\
(3)\end{array}$ & 0.023 \\
\hline
\end{tabular}

Table 4 Summary of all pairwise examinations for joint segregation of minisatellite DNA loci undertaken in brown trout family F89/1. X denotes pairwise comparisons analysed (male above, and female below the diagonal)

Segregation in males

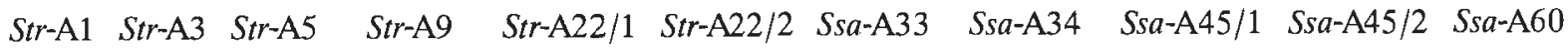

\begin{tabular}{|c|c|c|c|c|c|c|c|c|c|c|c|}
\hline \multicolumn{12}{|c|}{ Segregation in females } \\
\hline Str-A1 & & - & - & - & - & - & - & - & - & - & - \\
\hline Str-A3 & $x$ & & $x$ & $x$ & $x$ & $\mathrm{x}$ & $x$ & $x$ & - & $x$ & - \\
\hline Str-A5 & $x$ & $x$ & & $x$ & $x$ & $x$ & $x$ & $x$ & - & $x$ & - \\
\hline Str-A9 & $x$ & $\mathrm{x}$ & $x$ & & $x$ & $\mathrm{x}$ & $x$ & $x$ & - & $x$ & - \\
\hline Str-A22/1 & $x$ & $\mathrm{x}$ & $x$ & $x$ & & 0 & $x$ & $\mathrm{x}$ & - & $\mathrm{x}$ & - \\
\hline Str-A22/2 & $x$ & $x$ & $x$ & $x$ & 0 & & $x$ & $\mathrm{x}$ & - & $x$ & - \\
\hline Ssa-A33 & $\mathrm{x}$ & $\mathrm{x}$ & $\mathrm{x}$ & $x$ & $x$ & $\mathrm{x}$ & & $x$ & - & $x$ & - \\
\hline Ssa-A34 & $x$ & $x$ & $x$ & $x$ & $\mathrm{x}$ & $x$ & $x$ & & - & $x$ & - \\
\hline Ssa-A45/1 & $x$ & $\mathrm{x}$ & $x$ & $\mathrm{x}$ & $x$ & $x$ & $x$ & $\mathrm{x}$ & & - & - \\
\hline Ssa-A45/2 & $\mathrm{x}$ & $\mathrm{x}$ & $x$ & $\mathrm{x}$ & $x$ & $\mathrm{x}$ & $x$ & $\mathrm{x}$ & $\mathrm{x}$ & & - \\
\hline Ssa-A60 & $x$ & $x$ & $x$ & $x$ & $x$ & $x$ & $x$ & $x$ & $x$ & $x$ & \\
\hline
\end{tabular}

Statistically significant associations $(P<0.05)$ are indicated by $O$ s. 
segregation data for males and females must be considered separately since some linkage associations in salmonids can be male-specific, i.e. 'pseudolinkage' (see Wright et al., 1983 for details). Statistically significant (i.e. $P<0.05$ ) nonrandom assortment was detected in only two instances, involving both male and female comparisons of Str-A22/1 with Str-A22/2. These segregation data are detailed in Table 5. Tight linkage was observed, with no nonparental genotype observed among the 54 offspring screened. Inspection of progeny segregation from families $89 / 2$ and $89 / 3$ tends to confirm joint segregation of these loci, though numbers were considered too small to apply meaningful statistical analysis (data not shown).

\section{Discussion}

In general, single locus minisatellite probes have been isolated from species (e.g. humans, other mammals and birds) that produce relatively few offspring. Thus, with the exception of human studies (e.g. Nakamura et al., 1988; Armour et al., 1990) where many and extensive family pedigree samples have been available, linkage analyses have been limited by small progeny/family numbers. More fecund groups (e.g. fishes) provide an opportunity for more direct investigations of inheritance, linkage and mutation rates of minisatellite loci in large single generation families.

The single locus inheritance data confirm that at all 11 loci examined, specific minisatellite alleles segregated in Mendelian fashion. No novel allele (i.e. new mutation) was observed, although such alleles have been occasionally described in other pedigree analyses. For example, mutation rates as high as 0.05 per gamete have been reported for some human minisatellite loci (Jeffreys et al., 1988; Wolff et al., 1988). Similarly unstable minisatellite loci have been described in other species; mouse (Kelly et al., 1989) and willow warbler (Gyllensten et al., 1989). Given both the relatively small sample size screened in this study and the comparatively moderate levels of heterozygosity (0-69 per cent, with average of 54 per cent) exhibited by the minisatellite loci in brown trout populations, the absence of mutant alleles was not unexpected. In general, there is little detailed information available concerning the molecular mechanisms involved in the production of new alleles at minisatellite loci (Cohen, 1990; Chakraborty et al., 1991). Indeed, different mechanisms may apply to different minisatellites (e.g. Jeffreys et al., 1991; Wolff et al., 1991).

Following high stringency washings, all five Atlantic salmon derived minisatellite DNA probes also detected single locus variability in brown trout. Given such results, and because $S$. trutta and $S$. salar are congeneric, it is highly probable that the probes are detecting homologous loci in the two species. This has yet to be confirmed (e.g. by sequence analysis), as Gray \& Jeffreys (1991) have reported on an SLP from one species cross-hybridizing to a nonhomologous locus in other species. Similar cross-species hybridization of single locus minisatellite DNA probes has been observed in other studies, where detection of homologous loci was likewise considered probable but not proven conclusively (e.g. Gyllensten et al., 1990; Wolff et al., 1991; Hanotte et al., 1991a; Hanotte et al., 1992). Interestingly, in some cases, the fragment size range observed for a particular probe in the two salmonid species is strikingly different. For example, $\mathrm{p} S$ sa-A60 detects large fragments $(9-20 \mathrm{~kb})$ in Atlantic salmon populations (Taggart \& Ferguson 1990b; unpublished data) compared to $<0.5-2 \mathrm{~kb}$ in brown trout. Conversely, $\mathrm{pSsa}-\mathrm{A} 45 / 1$ detects a relatively small size range of fragments $(2-3 \mathrm{~kb})$ in Atlantic salmon (Taggart \& Ferguson, 1990b; unpublished data) but much larger fragments $(4.3-15 \mathrm{~kb})$ in brown trout. Further comparative investigations of these loci may provide information concerning possible molecular mechanisms governing size constraints of minisatellite DNA regions. Alternatively, other factors (e.g. speciesspecific RFLPs in the flanking regions, minisatellite

Table 5 Joint segregation analyses between Str-A22/1 and Str-A22/2 loci in the brown trout family F89/1

\begin{tabular}{|c|c|c|c|c|c|c|c|c|c|c|}
\hline & \multicolumn{2}{|c|}{ Parents } & \multirow{2}{*}{\multicolumn{4}{|c|}{ Progeny type }} & \multirow[b]{3}{*}{$N$} & \multirow[b]{3}{*}{$\chi_{\mathrm{AB}}^{2}$} & \multirow[b]{3}{*}{$P$} & \multirow[b]{3}{*}{$r$} \\
\hline & \multirow{2}{*}{$\begin{array}{c}\text { Locus A } \\
\text { Str-A22/1 }\end{array}$} & \multirow{2}{*}{$\begin{array}{l}\text { Locus B } \\
\text { Str-A22/2 }\end{array}$} & & & & & & & & \\
\hline & & & $a_{1}$ & $a_{2}$ & $a_{3}$ & $a_{4}$ & & & & \\
\hline$\frac{\text { Female }}{\text { Male }}$ & $\begin{array}{l}\mathrm{BC}\left(\mathrm{AA}^{\prime}\right) \\
\mathrm{AD}(\mathrm{AA})\end{array}$ & $\begin{array}{l}\mathrm{BC}\left(\mathrm{BB}^{\prime}\right) \\
\mathrm{AB}(\mathrm{BB})\end{array}$ & 0 & 25 & 29 & 0 & 54 & 54 & $<0.001$ & 0 \\
\hline $\begin{array}{l}\text { Female } \\
\text { Male }\end{array}$ & $\begin{array}{l}\mathrm{BC}(\mathrm{AA}) \\
\mathrm{AD}\left(\mathrm{AA}^{\prime}\right)\end{array}$ & $\begin{array}{c}\mathrm{BC}(\mathrm{BB}) \\
\mathrm{AB}\left(\mathrm{BB}^{\prime}\right)\end{array}$ & 29 & 0 & 0 & 25 & 54 & 54 & $<0.001$ & 0 \\
\hline
\end{tabular}

The informative parent is underlined in each analysis. Symbols as in text. 
clusters, possible nonhomology) could account for the observed differences.

Associations between phenotypic sex and biochemi$\mathrm{cal} /$ molecular markers in salmonids have been poorly studied to date. This has been at least partly due to the need for dedicated facilities for holding separate family groups to an age ( $>6$ months posthatching) where their sex can be confidently determined (May et al., 1989). Nevertheless, there have been a few examples of sex linkage with such markers. Gelman et al. (1987) have reported a linkage association between a locus encoding hexosaminidase and phenotypic sex in rainbow trout, while May et al. (1989) have demonstrated tight classical linkage between sex and three isozyme loci $\left(L D H-1^{*}, A A T-5^{*} \& G P I-3^{*}\right)$ in second generation spartics (Salvelinus fontinalis $\times S$. alpinus). More recently Devlin et al. (1991), using subtractive hybridization methodologies, have isolated a malespecific (i.e. Y chromosome) probe in chinook salmon (O. tshawytscha). Interestingly, the strength of hybridization signal obtained with this probe suggests that it is a repeated sequence that is being recognized in the male chinook.

The sex chromosomes of salmonids (in common with most other fish) are morphologically similar to autosomes and indeed have yet to be positively identified in the majority of species, including the brown trout (Hartley, 1987). Where identified (whether directly, by more sensitive cytological techniques, or indirectly, through sex reversal experimentation) the male has been consistently demonstrated as the heterogametic sex, e.g. Onchorhynchus mykiss (Thorgaard \& Allen, 1987; Chevassus et al., 1988), O. nerka (Thorgaard, 1978), O. kisutch (Hunter et al., 1982), Savelinus namaycush (Phillips \& Ihssen, 1985). The apparent male heterogametic condition detected by pStr-A9 in brown trout in this present study is thus in agreement with these findings. It should be feasible to locate physically the sex chromosomes of brown trout through in situ hybridization studies, though a larger probe than is currently available $(1.8 \mathrm{~kb})$ would probably be required. More detailed investigations, e.g. chromosome walking, could eventually lead to the isolation of both $\mathrm{X}$ - and Y-specific sequences and, hence, to a better understanding of sex determination in this and other salmonids. With hormonal manipulation of both sex and reproduction having become an important practice in aquaculture and fisheries biology, accurate methods of distinguishing between genetic and phenotypic sex in salmonid broodstock are in demand (Devlin et al., 1991). Rapid PCR-based methods of genotypic sex determination could be developed from known $\mathrm{X}$ and $\mathrm{Y}$ chromosome-specific sequences.
Associations between minisatellite loci and phenotypic sex have been reported in a few other studies. Analyses of multilocus DNA fingerprints have revealed sex-linked fragments in, for example, the laboratory mouse (Jeffreys et al., 1987) and parrots (Miyaki et al., 1992). May et al. (1993) have described a minisatellite DNA probe isolated from the red kite (Milvus milvus) which detects a multibanded fingerprint in females, yet does not hybridize to DNA from the male of the species. One of 23 human minisatellite loci isolated by Armour et al. (1990) was found to map to the $\mathrm{X}$ chromosome. Other types of repetitive DNA have also been linked to sex determining locations. For example Bkm satellite DNA, which contains numerous GATA-GACA repeats, is associated with sex determining chromosomes in snakes (Singh et al., 1980); mice (Jones \& Singh, 1982) and sea turtles (Demas et al., 1990). While Bkm sequences have been shown to detect DNA fingerprint patterns in both rainbow trout (Lloyd et al., 1989) and in the coral reef fish, Anthias squamipinnis (Wachtel \& Demas, 1991), no evidence of sex association was found in either study.

Most other studies of minisatellite loci in different species have shown them to be widely dispersed among (at least) the autosomes, even where only a single minisatellite probe has been used to generate DNA fingerprints (reviewed by Jeffreys et al., 1991). However, occasional occurrences of nonrandom associations (i.e. linkage) between minisatellite loci have been reported, e.g. in humans (Royle et al., 1988); cattle (Georges et al., 1990); birds (Hanotte et al., 1991b); Atlantic salmon (Taggart \& Ferguson, 1990a). This suggests the existence in the genome of these species and probably most others, of clusters of closely linked minisatellite loci (Royle et al., 1988). With brown trout having a relatively large chromosome complement $(2 n=80)$, the demonstration of random segregation among all of the independently isolated loci was not particularly surprising.

The linkage association detected between Str-A22/1 and Str-A22/2 was to be expected since both probes were derived from a single original recombinant. This linkage presents practical limitations in the use of both loci in population genetic studies, since independence of markers is a basic assumption of most statistical analyses commonly employed (Nei, 1978). Similarly, this knowledge is of obvious importance in determining the probability of relatedness within and among family groups. Even when washed to relatively low stringency $\left(1 \times \mathrm{SSC}, 65^{\circ} \mathrm{C}\right)$ neither $\mathrm{pStr}-\mathrm{A} 22 / 1$ nor $\mathrm{pStr}-\mathrm{A} 22 / 2$ detected the other linked locus, suggesting that the repetitive sequences of both probes are dissimilar. Rather than being the products of a single large minisatellite locus with a single $P a l$ site, therefore, both 
cloned fragments appear to contain distinct minisatellite loci and perhaps represent a 'minisatellite cluster' as mentioned above. It may be possible to follow the conservation of this minisatellite linkage group among other salmonid species, to gain further insight into both the mechanisms and rates of mutation at these loci (Wolff et al., 1991). There was no evidence of linkage between $S_{s a}-\mathrm{A} 45 / 1$ and $S s a-\mathrm{A} 45 / 2$ in brown trout despite both probes being derived from a single Atlantic salmon EMBL3 recombinant. However, these loci also show apparent independent segregation in Atlantic salmon (unpublished data). Whether the original insert DNA represented a contiguous length of DNA, or was the result of the ligation of three smaller unrelated fragments has yet to be ascertained.

The main purpose for our minisatellite DNA cloning studies was to obtain a series of highly polymorphic probes for use in detailed population genetic studies of native salmonids (Taggart \& Ferguson, 1990b; Prodöhl et al., 1994). All the probes described in this paper permit the unambiguous identification of (relatively stable) allelic variation at single loci and as such can be analysed similarly to, and combined with, allozyme and other nuclear data. However, it must be realised that at present, the collection of SLP data is much more time-consuming and costly than for allozyme screening and, as such, the application of minisatellite DNA-based studies should be carefully targeted. In future years it may prove possible to PCR amplify routinely some of these minisatellite loci, particularly the smaller ones, e.g. Str-A1, Str-A22/2 \& SsaA60. During our work many minisatellite DNA inserts were cloned which produced more complex fragment patterns than those described above (Prodöhl et al., 1994; unpublished results). While of little use in population studies, these probes may provide an additional source of chromosomal markers for further detailed linkage analyses.

\section{Acknowledgements}

We are grateful to Dr R. Hynes for useful discussion on preliminary drafts of the manuscript and to Dr C. Maggs for photographic assistance. The award of a Postgraduate Studentship to P.A.P. by the Brazilian Federal Agency of Postgraduate Education (CAPES - No. 487/89-5), and an advanced research fellowship to J.B.T. from the Natural Environmental Research Council are gratefully acknowledged.

\section{References}

ARMOUR, J. A. L., POVEY, S., JEREMIAH, S. AND JEFFREYS, A. J. 1990. Systematic cloning of human minisatellites from ordered array charomid libraries. Genomics, 8, 501-512.
BENTZEN, P., HARRIS, A. S. AND WRIGHT, J. M. 1991. Cloning of hypervariable minisatellite and simple sequence microsatellite repeats for DNA fingerprinting of important aquacultural species of salmonids and tilapias. In: Burke, T., Dolf, G., Jeffreys, A. J. and Wolff, R. (eds) DNA Fingerprinting: Approaches and Applications, pp. 243-262. Birkhäuser Verlag, Basel.

BRUFORD, M. W. AND BURKE, T. 1991. Hypervariable DNA markers and their applications in the chicken. In: Burke, T., Dolf, G., Jeffreys, A. J. and Wolff, R. (eds) DNA Fingerprinting: Approaches and Applications, pp. 230-242. Birkhäuser Verlag, Basel.

BURKE, T., HANOTTE, O., BRUFORD, M. W. AND CAIRNS, E. 1991. Multilocus and single locus minisatellite analysis in population biological studies. In: Burke, T., Dolf, G., Jeffreys, A. J. and Wolff, R. (eds) DNA Fingerprinting: Approaches and Applications, pp. 154-168. Birkhäuser Verlag, Basel.

CHAKRABORTY, R., FORNAGE, M., GUEGUEN, R. AND BOERWINKLE, E. 1991. Population genetics of hypervariable loci: analysis of PCR based VNTR polymorphism within a population. In: Burke, T., Dolf, G., Jeffreys, A. J, and Wolff, R. (eds) DNA Fingerprinting: Approaches and Applications, pp. 127-143. Birkhäuser Verlag, Basel.

Chevassus, B., DevauX, A., Chourrout, D. AND Jalabert, B. 1988. Production of YY rainbow trout males by self-fertilization of induced hermaphrodites. J. Hered., 79, 89-92.

COHEN, J. E. 1990. DNA fingerprinting for forensic identification: potential effects on data interpretation of subpopulation heterogeneity and band number variability. Am. J. Hum. Genet., 46, 358-368.

COPPIETERS, W., VAN DE WEGHE, A., DEPICKER, A., BOUQUET, Y. AND EVEREN, A. 1990. A hypervariable pig DNA fragment. Anim. Genet., 21, 29-38.

DAl.GLEISH, R. 1987. Southern blotting. In: Boulnois, G. J. (ed.) Gene Cloning and Analysis: A Laboratory Guide, pp. 45-60. Blackwell Scientific Publications, Oxford.

DEMAS, S., DURONSLET, M., S., W., CAILLOUET, C. AND NAKAMURA, D. 1990. Sex specific DNA in reptiles with temperature sex determination. J. Exp. Zool., 253, 319-324.

DEVLIN, R. H., McNEIL, B. K., GROVES, T. D. D. AND DONALDSON, E. M. 1991. Isolation of a Y-chromosomal DNA probe capable of determining genetic sex in Chinook salmon (Oncorhynchus tshawytscha). Can. J. Fish. Aquat. Sci., 48, 1606-1612.

FERGUSON, A. 1980. Biochemical Systematics and Evolution. Blackie, Glasgow.

FERGUSON, A. AND TAGGART, J. B. 1991. Genetic differentiation among the sympatric brown trout (Salmo trutta) populations of Lough Melvin, Ireland. Biol. J. Linn. Soc., 43, 221-237.

FIELDS, R. D., JOHNSON, K. R. AND THORGAARD, G. H. 1989. DNA fingerprints in rainbow trout detected by hybridization with DNA of bacteriophage M13. Trans. Am. Fish. Soc., $118,78-81$.

GElman, W. A., ALLENDORF, F. W. AND THORGAARD, G. H. 1987. Hexosaminidase is sex linked in rainbow trout. Isozyme Bull., 20, 14.

GEORGES, M., LATHROP, M., HILBERT, P., MARCOTTE, A., SCHWERS, A., SWILLENS, S., VASSART, G. AND HANSET, R. 1990. On the use of 
DNA fingerprints for linkage studies in cattle. Genomics, 6, 461-474.

GRAY, I. C. AND JEFFREYS, A. J. 1991. Evolutionary transience of hypervariable minisatellites in man and the primates. Proc. R. Soc. B., 243, 241-253.

GYLLENSTEN, U. B., JAKOBSSON, S., TEMRIN, H. AND WILSON, A. C. 1989. Nucleotide sequence and genomic organization of bird minisatellites. Nucl. Acids Res., 17, 2203-2214.

GYLLENSTEN, U. B., JAKOBSSON, S. AND TEMRIN, H. 1990. No evidence for illegitimate young in monogamous and polygynous warblers. Nature, 343, 168-170.

HANOTTE, O., BURKE, T., ARMOUR, J. A. L. AND JEFFREYS, A. J. 1991 a. Hypervariable minisatellite DNA sequences in the Indian peafowl Pavo christatus. Genomics, 9, 587-597.

HANOTTE, O., BURKE, T., ARMOUR, J. A. L. AND JEFFREYS, A. J. 1991 b. Cloning, characterization and evolution of Indian peafowl Pavo christatus minisatellite loci. In: Burke, T., Dolf, G., Jeffreys, A. J. and Wolff, R. (eds) DNA Fingerprinting: Approaches and Applications, pp. 193-216. Birkhäuser Verlag, Basel.

HANOTTE, O., BRUFORD, M. W. AND BURKE, T. 1992. Multilocus DNA fingerprints in gallinaceous birds: general approach and problems. Heredity, 68, 481-494.

HARTLEY, S. E. 1987. The chromosomes of salmonid fishes. Biol. Rev., 62, 197-214.

HUNTER, G. A., DONALDSON, E. M., GOETZ, F. W. AND EDGELL, P. R. 1982. Production of a female and sterile Coho salmon, and experimental evidence for male heterogamety. Trans. Am. Fish. Soc., 111, 367-372.

JEFFREYS, A. J., WILSON, V., KELLY, R., TAYLOR, B. A. AND BULFIELD, G. 1987. Mouse DNA 'fingerprints': analysis of chromosome localizations and germ-line stability of hypervariable loci in recombinant inbred strains. Nucl. Acids Res., 15, 2823-2837.

JEFFREYS, A. J., ROYLE, N., WILSON, v. AND WONG, Z. 1988. Spontaneous mutation rates to new length alleles at tandem-repetitive hypervariable loci in human DNA. Nature, 332, 278-281.

JEFFREYS, A. J., ROYLE, N. J., PATEL, 1., ARMOUR, J. A. L., MACLEOD, A., COLLICK, A., GRAY, I. C., NEUMANN, R., GIBBS, M., CROSIER, M., HILL, M., SIGNER, E. AND MONCKTON, D. 1991. Principles and recent advances in human DNA fingerprinting. In: Burke, T., Dolf, G., Jeffreys, A. J. and Wolf, R. (eds) DNA Fingerprinting: Approaches and Applications, pp. 1-19. Birkhäuser Verlag, Basel.

JONES, K. W. AND SINGH, L. 1982. Conserved sex-associated repeated DNA sequences in vertebrates. In: Dover, G. A. and Flavell, R. B. (eds) Genome Evolution, pp. 135-154. Academic Press, New York.

KELLY, R., BULFIELD, G., COLLICK, A., GIBBS, M. AND JEFFREYS, A. J. 1989. Characterization of a highly unstable mouse minisatellite locus: evidence for somatic mutation during early development. Genomics, 5, 844-856.

KOMINAMI, R., MITANI, K. AND MURAMATSU, M. 1988. Nucleotide sequence of a mouse minisatellite DNA. Nucl. Acids Res., $16,1197$.

LLOYD, M. A., FIELDS, M. J. AND THORGAARD, G. H. 1989. BKm minisatellite sequences are not sex associated but reveal DNA fingerprint polymorphisms in rainbow trout. Geno$m e, 32,865-868$.
MATHER, K. 1951. The Measurement of Linkage in Heredity. Methuen, London.

MAY, B., WRIGHT, J. E. AND STONEKING, M. 1979. Joint segregation of biochemical loci in Salmonidae: Results from experiments with Salvelinus and review of the literature on other species. Can. J. Fish. Aquat. Sci., 36, 1114-1128.

MAY, B., JOHNSON, K. R. AND WRIGHT, J. E. 1989. Sex linkage in Salmonids: Evidence from a hybridized genome of Brook trout and Arctic charr. Biochem. Genet., 27, 291-301.

MAY, C. A., WETTON, J. H. AND PARKIN, D. T. 1993. Polymorphic sex-specific sequences in birds of prey. Proc. R. Soc. B, 253, 271-276.

MIYAKI, C. Y., HANOTTE, O., WAJNTAL, A. AND BURKE, T. 1992. Sex-typing of Aratinga parrots using the human minisatellite probe 33.15. Nucl. Acids Res., 20, 5235-5236.

NAKAMURA, Y., LEPPERT, M. O'CONNELL, P., WOLFF, R., HOLM, T., CULVER, M., MARTIN, C., FUIIMOTO, E., HOFF, M., KUMLIN, E. AND WHITE, R. 1987. Variable number of tandem repeat (VNTR) markers for human gene mapping. Science, 235, $1616-1622$.

NAKAMURA, Y., LATHROP, M., O'CONNELL, P., LEPPERT, M., LALOUEL, J. M. AND WHITE, R. 1988. A primary map of ten DNA markers and two serological markers for human chromosome 19. Genomics, 3, 67-71.

NEI, M. 1978. Estimation of average heterozygosity and genetic distance from a small number of individuals. Genetics, 89, 583-590.

OHNO, S. M., MURAmoto, J., KLEIN, J. AND ATKIN, N. B. 1969. Diploid-tetraploid relationship in clupeoid and salmonoid fishes. In: Darlington, C. D. and Lewis, K. R. (eds) Chromosomes Today, pp. 139-147. Oliver and Boyd, Edinburgh.

PHILLIPS, R. B. AND IHSSEN, P. E. 1985. Identification of sex chromosomes in lake trout (Salvelinus namaycush). Cytogenet. Cell Genet., 39, 14.

PRODÖHL, P. A., TAGGART, J. B. AND FERGUSON, A. 1992. Genetic variability within and among sympatric brown trout (Salmo trutta) populations: multi-locus DNA fingerprint analysis. Hereditas, 117, 45-50.

PRODÖHL, P. A., TAGGART, J. B. AND FERGUSON, A. 1994. Cloning of highly variable minisatellite DNA single locus probes for brown trout (Salmo trutta L.) from a phagemid library. In: Beaumont, A. R. (ed.) Genetics and Evolution of Aquatic Organisms, pp. 263-270. Chapman and Hall, London.

ROYLE, N. J., CLARKSON, R. E., WONG, Z. AND JEFFREYS, A. J. 1988. Clustering of hypervariable minisatellites in the proterminal regions of human autosomes. Genomics, 3, 352-360.

RYMAN, N. 1991. Conservation genetics considerations in fishery management. J. Fish Biol., 39, 211-214.

SHAKLEE, J. B., KLAYBOR, D. C., YOUNG, S. AND WHITE, B. A. 1991. Genetic stock structure of odd-year pink salmon, Oncorhynchus gorbuscha (Walbaum), from Washington and British Columbia and potential mixed-stock fisheries applications. J. Fish Biol., 39 (Suppl. A), 21-34.

SINGH, L., PURDOM, I. F. AND JONES, K. W. 1980. Sex chromosome associated satellite DNA: evolution and conservation. Chromosoma, 79, 137-157.

TAGGART, J. B. AND FERGUSON, A. 1984. Allozyme variation in the brown trout (Salmo trutta L.): single locus and joint 
segregation inheritance studies. Heredity, 53, 339-359.

TAGGART, J. B. AND FERGUSON, A. 1990a. Minisatellite DNA fingerprints of salmonid fishes. Anim. Genet., 21, 377-389.

TAGGART, J. B. AND FERGUSON, A. 1990b. Hypervariable minisatellite DNA single locus probes for the Atlantic salmon, Salmo salar L. J. Fish Biol., 37, 991-993.

TAGgart, J. B., HYNES, R. A., PRODÖHL, P. A. AND FERGUSON, A. 1992. A simplified protocol for routine total DNA isolation from salmonid fishes. J. Fish Biol., 40, 963-965.

TAGGART, J. B., PRODÖHL, P. A. AND FERGUSON, A. 1994. Genetic markers for Atlantic salmon (Salmo salar L.): Single locus inheritance and joint segregation analyses of minisatellite (VNTR) DNA loci. Anim. Genet., (in press).

THORGAARD, G. H. 1978. Sex chromosomes in the Sockeye salmon: a Y-autosome fusion. Can. J. Genet. Cytol., 20, 349-354.

THORGAARD, G. H. AND ALLEN, S. K. 1987. Chromosome manipulation and markers in fishery management. In: Ryman, N. and Utter, F. (eds) Population Genetics and Fishery Management, pp. 319-332. University of Washington Press, London.

UTTER, F. M. 1991. Biochemical genetics and fishery management: an historical perspective. J. Fish Biol., 39 (Suppl. A), $1-20$.

VERSPOOR, E. 1994. The evolution of genetic divergence at protein coding loci among anadromous and nonanadro- mous populations of Atlantic salmon Salmo salar. In: Beaumont, A. R. (ed) Genetics and Evolution of Aquatic Organisms, pp. 52-67. Chapman and Hall, London.

WACHTEL, s. AND DEMAs, s. 1991. Bkm satellite DNA and $Z F Y$ in the coral reef fish Anthias squamipinnis. Genome, 34, 612-617.

WOLFF, R., NAKAMURA, Y, AND WHITE, R. 1988. Molecular characterization of a spontaneously generated new allele at a VNTR locus: no exchange of flanking DNA sequence. Genomics, 3, 347-351.

WOLFF, R., NAKAMURA, Y., ODELBERG, S., SHIANG, R. AND WHITE, R. 1991. Generation of variability at VNTR loci in human DNA. In: Burke, T., Dolf, G., Jeffreys, A. J. and Wolff, R. (eds) DNA Fingerprinting: Approaches and Applications, pp. 20-38. Birkhäuser Verlag, Basel.

WONG, Z., WILSON, V., PATEL, I., POVEY, S. AND JEFFREYS, A. J. 1987. Characterization of a panel of highly variable minisatellites cloned from human DNA. Ann. Hum. Genet., 51, 269-288.

WRIGHT, J. E., JOHNSON, K, HOLLISTER, A. AND MAY, B. 1983. Meiotic models to explain classical linkage, pseudolinkage, and chromosome pairing in tetraploid derivative Salmonid genomes. In: Rattazzi, M. C., Scandalios, J. G. and Whitt, G. S. (eds) Isozymes: Current Topics in Biological and Medical Research, pp. 239-260. Alan R. Liss, New York. 